\title{
Biomarkers for early diagnosis, prognosis, prediction, and recurrence monitoring of non- small cell lung cancer
}

This article was published in the following Dove Press journal:

OncoTargets and Therapy

12 September 2017

Number of times this article has been viewed

\author{
Yong Tang $1,2, *$ \\ Guibin Qiaol,2,* \\ Enwu $X u^{2}$ \\ Yiwen Xuan ${ }^{2}$ \\ Ming Liao ${ }^{2}$ \\ Guilin Yin' \\ 'Southern Medical University, \\ Guangzhou, Guangdong Province, \\ China, ${ }^{2}$ Department of Thoracic \\ Surgery, General Hospital of \\ Guangzhou Military Command of \\ PLA, Yuexiu District, Guangzhou City, \\ Guangdong Province, China \\ *These authors contributed equally \\ to this work
}

Correspondence: Guilin Yin; Yong Tang Southern Medical University, No 1023 , South Shatai Road, Guangzhou 510515 , Guangdong Province, China Email yingI1952@I63.com; ywzIII2@sina.com

\begin{abstract}
Despite advances in the management of non-small cell lung cancer, it remains to be the leading cause of cancer-related deaths worldwide primarily because of diagnosis at a late stage with an overall 5-year survival rate of $17 \%$. A reduction in mortality was achieved by low-dose computed tomography screening of high-risk patients. However, the benefit was later challenged by the high false positive rate, resulting in unnecessary follow-ups, thus entailing a burden on both the health care system and the individual. The diagnostic dilemma imposed by imaging modalities has created a need for the development of biomarkers capable of differentiating benign nodules from malignant ones. In the past decade, with the advancements in high-throughput profiling technologies, a huge amount of work has been done to derive biomarkers to supplement clinical diagnosis. However, only a few of them have efficient sensitivity and specificity to be utilized in clinical settings. Therefore, there is an urgent need for the development of sensitive and specific means to detect and diagnose lung cancers at an early stage, when curative interventions are still possible. Due to the invasiveness of tissue biopsies and inability to capture tumor heterogeneity, nowadays enormous efforts have been invested in the development of technologies and biomarkers that enable sensitive and cost-effective testing using substrates that can be obtained in a noninvasive manner. This review, primarily focusing on liquid biopsy, summarizes all documented potential biomarkers for diagnosis, monitoring recurrence treatment response.
\end{abstract}

Keywords: liquid biopsy, circulating-tumor DNA, circulating tumor cells, non-small cell lung cancer, exosomes, biomarkers

\section{Introduction}

Despite the progress made in the treatment of non-small cell lung cancer (NSCLC) over the past few decades, the 5-year survival rate has not improved much primarily because of initial diagnosis at a late stage. Currently, its detection primarily relies on computed tomography (CT) scans in combination with sputum cytology test. The 5-year survival rate, ranging from $50 \%$ for stage IA to $2 \%$ for stage IV, improves significantly when diagnosed at an early stage. ${ }^{1}$ However, only one third of patients with NSCLC are diagnosed at an early stage when the disease is still localized and potentially curable. Most cases are diagnosed with locally advanced or metastatic disease. ${ }^{2}$

Lung cancer screening aiming to detect malignant nodules at an early stage has been applied to improve the survival rate. Recent implementation of low-dose computed tomography (LDCT) screening has been shown to diagnose lung cancer at an earlier stage with survival benefits, and therefore, it is recommended to perform on healthy individuals with high risk, such as current and former smokers. ${ }^{3}$ LDCT has 
been shown to double the early detection rate (from $30 \%$ to $60 \%$ ) and reduce mortality rate by $20 \%$ comparing to chest radiography. ${ }^{3-5}$ However, the reduction in mortality was challenged by a significant increase in false positives, resulting in unnecessary follow-ups, thus entailing a burden on the health care system and the individual. ${ }^{6-8}$ National lung screening trial ${ }^{3}$ revealed that in order to prevent one case of lung cancer-related mortality, 320 individuals with high risk need to undergo LDCT. Therefore, there is an urgent need to derive biomarkers to better stratify high-risk individuals, thus maximizing the benefits from LDCT screening.

In addition to LDCT, many biomarkers, especially molecular biomarkers, were developed to supplement clinical diagnosis. In the past few decades, researchers explored tumor-specific alterations primarily by tissue biopsies to interrogate the genome and epigenome of NSCLC to derive biomarkers for diagnosis, prognosis, and monitoring. However, tumor biopsy is not only invasive but also biased with limited ability to reflect tumor heterogeneity, owing to its temporal and spatial snapshot nature. Liquid biopsy has received enormous attention in the past few years owing to obvious clinical advantages. ${ }^{9,10}$ Circulating tumor DNA (ctDNA), composed of small fragments of nucleic acid released from apoptotic or necrotic tumor cells, can reflect the genetic profile of a tumor. ${ }^{11,12}$ Numerous studies have shown that ctDNA can be used as a surrogate for patient stratification, diagnosis, and disease monitoring. ${ }^{13}$ Nowadays, with improved understanding of molecular features associated with NSCLC and the availability of new technologies that have enabled sensitive and specific biomarker analysis using liquid biopsy samples, it is becoming promising to use biomarkers detected in blood and/ or other types of body fluids for NSCLC diagnosis, treatment guidance, prognosis prediction, and recurrent monitoring.

A large variety of substrates in the circulation can be obtained from liquid biopsy samples and used for biomarker analysis, such as circulating tumor cells (CTCs), ctDNA, microRNA (miRNA), and serum proteins. With the efficacies achieved by immune checkpoint inhibitors, immune profiling of peripheral blood would also provide important biomarkers to predict and monitor patient response to immune modulators. This article reviews all documented biomarkers for detection and monitoring of NSCLC using blood samples.

\section{Circulating tumor cell}

The presence of CTCs was first discovered in 1896. During tumor formation and growth, cancerous cells are released into the blood stream. Such cells provide unprecedented insights into both primary and metastatic tumors. Numerous studies have shown that CTCs can serve as a biomarker for predicting disease progression and survival. ${ }^{14}$ High CTC numbers are often associated with aggressive disease, increased metastasis, and shorter disease-free survival (DFS). ${ }^{15}$ Collecting CTCs from blood is noninvasive; therefore, CTCs can be used for "real-time" evaluation of tumor dynamics. In addition, CTCs have the potential to guide treatment, reflect therapeutic efficacy, and reveal drug resistances. ${ }^{16}$

A number of CTC capturing techniques have been developed to concentrate and isolate the relatively rare cell population from whole blood. Two main principles have been employed based on either immunoselection in a fluid flow chamber or size separation by a filtration device. The former usually incorporates epithelial cell adhesion molecule (EpCAM)-positive selection system in which CTCs are selected by anti-EpCAM antibodies. ${ }^{17,18}$ To date, the CellSearch system based on this principle is the only USA Food and Drug Administration-approved CTC detection method to numerate CTC for limited tumor prognosis applications, but this system is not yet approved for NSCLC diagnosis. ${ }^{19-21}$ Incidence of CTCs in NSCLC was lower compared with other tumors as reported for the CellSearch method. ${ }^{19}$ In addition, EpCAM-positive CTCs are less frequently detected in NSCLC comparing to other epithelial tumors using different CTC detection methods, ${ }^{22}$ primarily because of epithelial-to-mesenchymal transition (EMT). Epithelial proteins have been shown to be downregulated in CTCs; therefore, these EMT-associated CTCs can be missed by EpCAM-based enrichment technologies. ${ }^{23}$ Isolation by size of epithelial tumor cells (ISET) is another method to separate CTC from leukocytes. Cancer cells are generally larger than normal cells, and even small-cell lung cancer cells are larger than circulating lymphocytes or large monocytes. Rarecells and ScreenCell systems are two devices available based on size difference, and both of them use a filter with particular pore size to capture individual and viable CTC. ${ }^{24}$

CTCs can be detected in 20\%-30\% advanced NSCLC patients with metastasis, and the detection rate is associated with a positive lymph node status. ${ }^{25-28}$ However, the detection rate of CTCs in nonmetastatic patients is very low, suggesting that alternative methods should be utilized for early-stage patients. Multiple markers may also need to be incorporated in CTC capturing to increase the sensitivity of CTC detection in NSCLC patients.

\section{CTC quantification as a biomarker for prognosis, prediction, and treatment response assessment}

A study using the CellSearch method showed that 21 out of 101 late-stage treatment-naïve patients (stages III-IV) had at 
least 2 CTCs detected. The number of CTCs detected prior to and during treatment was correlated with overall survival (OS) and stage of disease. ${ }^{29}$ Hofman et al reported a study using ISET. All enrolled NSCLC patients underwent surgery and all stages were included, in which $36 \%$ were considered to be CTC-positive. Patients with high level ( $\geq 50$ cells, 31\% of all samples) of suspicious cells had a worse prognosis. ${ }^{30}$

Studies have shown that patients with an increased number of CTCs during treatment are often associated with radiographic tumor progression; in contrast, patients with a reduction in number of CTCs are associated with radiographic response. ${ }^{31}$ In patients with stage IV NSCLC, a correlation between changes in number of CTC and fluorodeoxyglucose-positron emission tomography or RECIST (Response Evaluation Criteria in Solid Tumors) response was observed. ${ }^{32}$ Patients harboring EGFR mutations were more likely to be CTC-positive compared to patients with wild-type $E G F R .^{22}$

Molecular changes in CTCs are thought to play important roles in response assessment. Currently, analysis of isolated CTCs can stratify patients according to their mutation types, such as EGFR, HER2, and KRAS mutations. ${ }^{33-35}$ In subsets of patients, CTCs were found to express genes involved in resistance to therapy such as HER3 and MET. Another study reported EGFR T790M detection in CTCs in 2 out of $6(33 \%)$ NSCLC patients who responded to tyrosine kinase inhibitor therapy compared with 9 out of 14 (64\%) with clinical progression. ${ }^{31}$

\section{Genomic rearrangement identified in CTCs}

CTC is potentially a noninvasive alternative to tissue biopsies and a surrogate for the entire tumor genome. Current progress in developing whole-genome amplification and whole transcriptome library construction made it possible for the sequencing-based analyses of CTCs to be scaled down to single-cell level.

Whole-genome analysis for CTCs was published by many labs. One NSCLC study isolated CTCs from seven lung adenocarcinoma patients using CellSearch System and performed whole-exome sequencing on each single CTC to detect single-nucleotide variations (SNVs) and copy number variations. The study reported that SNVs detected in single CTCs are highly heterogeneous, and that the important mutations associated with drug target $(E G F R)$, drug resistance $(P I K 3 C A)$, and phenotypic transition $(T P 53, R B 1)$ can all be detected in CTCs. ${ }^{36}$

CTCs can be separated according to different surface markers and physical properties using several methods, including flow cytometry and micromanipulation. However, due to their rareness in patient blood, especially for NSCLC, the number of CTCs is usually not sufficient to provide enough DNA or RNA for next-generation sequencing. Although the significant clinical value of CTC genomic analyses is obvious, the use of CTCs to obtain tumor genomic information needs further improvement in the identification of CTC, efficient isolation and capturing of viable cells, automated method for subsequent assessment of the retained cells, and standardized pipeline for sequencing data analysis before clinical implementation in NSCLC.

\section{Tumor-derived nuclear acids}

Tremendous efforts have been invested to explore the potential of ctDNA in diagnosing and monitoring treatment responses. Several studies have shown that increased levels of DNA fragments are found in the blood of cancer patients. ${ }^{37,38}$ Circulating free DNA (cfDNA) are small double-stranded fragments of DNA found in plasma. cfDNA is originating from different tissues, and hematopoietic cells are the main source in healthy subjects. ${ }^{39}$ Although with great variations among individuals, the concentration of cfDNA is usually very low - often $<10 \mathrm{ng}$ per $\mathrm{mL}$ of plasma. ${ }^{40,41}$ Increased level of cfDNA was often detected as a result of tissue trauma, inflammation, or diseases such as cancer. In the context of cancer, ctDNA, constituting a substantial part of cfDNA, primarily derives from apoptosis and necrosis of the cancer cells. ${ }^{37}$ Some studies have also reported alternative sources of ctDNA, such as CTCs ${ }^{42,43}$ and exosomes, which can be actively secreted by tumor cells. ${ }^{44,45}$ As the concentration of ctDNA is significantly affected by tumor volume, localization and vascularization as well as a patient's response to treatments, and hepatic and renal clearance function, the abundance of ctDNA in the blood may reflect tumor burden and provide an earlier indication of drug treatment response compared to imaging monitoring. ${ }^{46}$ The concordance rate between the genetic profiles of ctDNA and the corresponding tumor tissue has been demonstrated by numerous studies. ${ }^{47-49}$ Therefore, ctDNA is a valuable tool for mutation detection in cancer patients who cannot undergo tissue biopsy. However, challenges remain because ctDNA only represents a very small fraction of cfDNA, ${ }^{50,51}$ thus requiring methods with very high sensitivity.

\section{ctDNA as prognosis predictive marker}

Numerous studies have demonstrated the prognostic and predictive value of ctDNA in a number of cancers, including but not limited to NSCLC, colon cancer, breast cancer, and other malignancies. ${ }^{13,52,53}$ A pooled analysis including 
705 patients revealed a longer progression-free survival (PFS) observed in patients with EGFR mutation detected from ctDNA. ${ }^{54}$ Other studies have reported inferior PFS and OS associated with KRAS mutations detected in ctDNA in advanced NSCLC patients treated with chemotherapy. ${ }^{55}$ One of the most promising applications of ctDNA is its ability in detecting minimal residual disease after the implementation of curative therapies. Numerous studies have reported an inverse correlation between residual ctDNA and OS/DFS. ${ }^{56}$ In addition to specific mutations detected from ctDNA, the predictive power of changes in ctDNA upon pharmacological interventions was also investigated. A few studies have reported that the reduction in ctDNA can predict treatment response and OS. ${ }^{57}$

\section{Methylated DNA}

DNA methylation is rising as a promising marker for early detection, prognosis, and real-time follow-up of tumor dynamics. Hypermethylation of the $\mathrm{CpG}$ islands in promoter regions of tumor-suppressor genes has been shown to contribute to carcinogenesis. ${ }^{58}$ A number of loci have been identified in lung cancer that show significantly elevated DNA methylation in tumor tissue. ${ }^{59}$ In the plasma of lung cancer patients, DNA hypermethylation was also reported, including p16INK4A, DAPK11, RASSF1A, RAR, MGMT, GSTP1, and $A P C .{ }^{60}$ However, only $49 \%$ sensitivity and $85 \%$ specificity were achieved in identifying lung cancer patient from nonmalignant pulmonary disease. Several studies have reported the potential of investigating tumor-specific methylations in blood for screening and diagnosis of lung cancer. For example, various gene promoters were found to be differentially methylated in ctDNA between patients with lung cancer and controls, including short stature homeobox 2 (SHOX2), ${ }^{48,61}$ doublecortin like kinase 1 (DCLK1), ${ }^{62}$ septin9 (SEPT9), ${ }^{63}$ ras association domain family 1 isoform $\mathrm{A}(R A S S F 1 A)$, and retinoic acid receptor $\mathrm{B} 2$ (RARB2). ${ }^{64}$

DNA methylation can also be used as a prognostic biomarker. Detection of methylated breast cancer metastasis suppressor-1 (BRMS1) and (sex determining region Y)-box 17 (SOX17) in operable and advanced NSCLC was shown to have a negative impact on survival. ${ }^{47,65}$ In contrast, $S F N$ (14-3-3 Sigma) promoter methylation was correlated with a reduced risk of death. ${ }^{46}$ Interestingly, in addition to its diagnostic value, DCLK1 methylation was also associated with shorter survival. ${ }^{62}$

Several studies have reported the use of tumor-specific methylation for tracking a patient's response to therapy. Elevated level of $A P C$ and $R A S S F 1 A$ promoter methylation in ctDNA within $24 \mathrm{~h}$ after cisplatin-based therapy was reported, consistent with chemotherapy-induced cell death. ${ }^{66}$ Methylation of $S H O X 2, R A S S F 1 A$, and RARB2 has shown potential to monitor disease recurrence after surgery and chemotherapy. ${ }^{64,67}$ Patients with unmethylated checkpoint with forkhead and ring finger domains $(C H F R)$ promoter survived longer when receiving EGFR tyrosine kinase inhibitors as second-line treatment, compared to conventional chemotherapy. ${ }^{68}$

\section{MicroRNA}

It has been demonstrated that circulating miRNAs' levels in human plasma remain stable even when plasma is subjected to prolonged incubation at room temperature. Circulating miRNAs are also shown resistant to multiple freeze-thaw cycles. ${ }^{69}$ The noninvasiveness and stability make circulating miRNAs a potential tool to identify diagnostic markers in oncology. Indeed miRNA level changes were identified in certain tumor mouse models and lung cancer patients. ${ }^{69,70}$ It is, therefore, possible that cell-free miRNA signatures can be used to diagnose cancers.

Ever since miRNAs were identified in human plasma, many studies have been aiming at identifying a reliable diagnostic tool by investigating the differential expression of circulating miRNAs among NSCLC patients, healthy controls, and patients harboring benign tumors. It is found that compared to healthy donors, the expression of miR-25 is fivefold higher and miR-223 is threefold higher in serum of NSCLC patients. ${ }^{70}$ It has been demonstrated that a predictive model consisting of serum miR-15b-5p, miR-16-5p, and $\mathrm{miR}-20 \mathrm{a}-5 \mathrm{p}$ can be used to differentiate early-stage NSCLC cases from healthy subjects. ${ }^{71}$ Downregulation of miR-486-5p was observed in both primary tumors and the serum of NSCLC patients by several independent research groups, which suggests its potential as a tumor suppressor involved in multiple signaling circuits in NSCLC. ${ }^{72-74}$ Furthermore, a 34-miRNA panel can identify patients with early-stage NSCLC in a group of asymptomatic high-risk subjects with up to $80 \%$ accuracy. ${ }^{75}$ The signature could possibly serve as a noninvasive screening tool to stratify individuals at high risk. Most importantly, such signature is lung cancer specific. Moreover, an eight-miRNA panel was identified using three different analytical methods, which can be used for differentiating cancerous tissues from noncancerous ones in NSCLC patients. However, only three of these miRNAs (miR-30e-5p, miR-21, and miR-10a) are differentially expressed in NSCLC plasma specimens compared to plasma obtained from healthy individuals. ${ }^{76}$ 
Some miRNAs have even shown great potential as a therapeutic target of lung cancer. For instance, miR-21 is commonly overexpressed in cancers including lung, breast, and colorectal cancer (CRC) ${ }^{77}$ Higher expression of miR-21 and miR-155 in both tumor tissues and serum can predict recurrence and poor survival in NSCLC, which may suggest that overexpression of circulating miR-21 has great prognostic potential in NSCLC. ${ }^{78} \mathrm{miR}-21$ affects response to chemotherapy in several tumor types and thus can also act as a therapeutic target for overcoming drug resistance in cancers. Indeed, recent study has revealed that antisense inhibition of miR-21 or miR-221 sensitizes the effects of gemcitabine, a chemotherapeutic treatment of pancreatic cancer. ${ }^{71}$ Besides miR-21, let-7 also acts as a promising therapeutic target for lung cancer. Systemic delivery of synthetic let-7 mimic or miR-34a into lung tumor-bearing mice results in significant tumor regression compared to the delivery of an miRNA control. ${ }^{79}$ As most studies of miR-21 and let-7 are focusing on tumor tissues, the application of such circulating miRNA as a biomarker is still at research stage. Further validations are needed before applying them as biomarkers for the early diagnosis of cancers including NSCLC.

However, the inconsistency and even contradictions shown in different studies of miRNA evaluation make this method less applicable in clinical settings. This may be contributed by three factors: 1) the internal control gene used for the normalization of miRNA expression may vary between different research groups. Unfortunately, the field has not reached a uniform and reliable internal control for data analysis. 2) Circulating miRNAs do not exist only in exosomes, and most of them are shown to be associated with protein complexes; therefore, different materials such as plasma/serum, whole blood, or exosomes used for cell-free miRNAs isolation make the quality and quantity of circulating miRNAs isolated by different research groups vary. 3) In some studies, patient number is very limited, which may cause the results to be less reliable.

\section{Exosome as surrogate for mutation detection}

Exosomes are nanovesicles of $50-100 \mathrm{~nm}$ diameters that are released from most viable cells. Current knowledge suggests that exosomes form by membrane invagination of late endosomes, resulting in vesicles that contain cytosolic components and expose the extracellular domain of some plasma membrane receptors at their surface. ${ }^{80,81}$ Because of their cellular origin, exosomes bear specific protein markers of the endosomal pathway, such as tetraspanins (CD63, CD9, and CD81), heat shock proteins (HSP70), and proteins from the Rab family, Tsg101 and Alix, which are not found in other types of vesicles of similar size. ${ }^{82,83}$ Exosomes have been found in various body fluids of healthy individuals and patients with malignancies as cargos containing a wide variety of components such as single-stranded RNA, long noncoding RNA, miRNA, and double-stranded DNA. ${ }^{84}$ Profiling of exosomes provided tailored information of individual patients and may be crucial for a precise prognosis or a differential diagnosis.

Numerous studies have investigated and confirmed the potential of exosomes as surrogate for mutation detection. Double-stranded genomic DNA with mutated KRAS and $\mathrm{p} 53$ were identified in the serum exosomes of patients with pancreatic cancer. ${ }^{85}$ In addition, using whole-genome sequencing revealed that serum exosomes from patients with pancreatic cancer contain genomic DNA spanning all chromosomes. These results indicated that serum-derived exosomes can be used to determine genomic DNA mutations for cancer prediction, treatment, and therapy resistance. In an independent study, high prevalence of mutant $K R A S$ in circulating exosome-derived DNA (exoDNA) was detected in plasma of early-stage pancreatic cancer patients, by droplet digital PCR on exoDNA and cfDNA. ${ }^{86}$ KRAS mutations in exoDNA were identified in $7.4 \%, 66.7 \%, 80 \%$, and $85 \%$ of age-matched controls, localized, locally advanced, and metastatic pancreatic ductal adenocarcinoma (PDAC) patients, respectively. Although a higher percentage of patients with localized PDAC exhibited detectable KRAS mutations in exoDNA than previously reported for cfDNA, a substantial minority of healthy samples demonstrated mutant $K R A S$ in circulation, dictating careful consideration and application of liquid biopsy findings. In CRC, genomic DNA extracted from the tumor tissues of 35 patients with histologically confirmed CRC and exosomal mRNA obtained from peripheral blood of the corresponding patients prior to surgery are studied together. ${ }^{87}$ The KRAS mutation rates in tumor tissues and the matched serum exosomes were $57.6 \%$ and $42.4 \%$, respectively; $B R A F$ mutation was $24.2 \%$ and $18.2 \%$, respectively. There was no significant difference between the rate detected by tissue and serum exosomes. The total consistency rate was $94.9 \%$ and $93.9 \%$ for $K R A S$ and $B R A F$ mutations, respectively. These results suggested that serum exosomal mRNA may be used as a novel source for the rapid and noninvasive genotyping of cancer patients. ${ }^{88,89}$ Recently, EGFR T790M resistance mutation in lung cancer patients is successfully detected using exosomal RNA and the sensitivity is improved compared to analysis of ctDNA alone. ${ }^{90}$ 
Recent studies also suggest that plasma exosomes might be valuable diagnostic indicators in lung cancer. Profiling of the exosome proteins from the plasma of 219 suspected lung cancer patients with 37 antibodies targeting lung-cancerrelated proteins identified a 30-marker model classifying correctly $75 \%$ of patients (sensitivity of 0.75 and specificity of 0.76). ${ }^{91}$ Similarly, analysis of the plasma exosomes from 581 patients (431 with lung cancer and 150 control individuals) using a custom array (49 antibodies). The authors demonstrated that CDC151, CDC171, and tetraspanin 8 were the strongest discriminators of malignancy, compared with healthy controls, and proposed a 10-marker model as a diagnostic tool. ${ }^{92}$

\section{Serum protein markers}

NSCLC is often associated with the differential expression of several proteins, which may be potential biomarkers for lung cancer. For instance, carcinoembryonic antigen, CYFRA 21-1 (cytokeratine 19 fragment), neuron-specific enolase, progastrin-releasing peptide, and squamous cell carcinoma antigen are reported to be elevated in serum of a fraction of lung cancer patients. However, these protein markers are not sensitive or specific enough to detect lung cancer in a clinically relevant way or to have enough value as biomarker for the diagnosis of asymptomatic patients with lung cancer. ${ }^{93,94}$

\section{Summary}

Diagnosing NSCLC at an early stage remains to be a hurdle to improve the OS rate, which has only increased by a few percent with all the advancements in targeted therapies. Liquid biopsy, providing an opportunity for interrogating the cancer genome and epigenome noninvasively, allows for real-time tracking of tumor dynamics. The above-mentioned substrates in the blood have the potential to serve as early diagnosis, treatment stratification, and recurrent monitoring tools. While recent studies have produced numerous promising biomarkers, translating such markers to the bedside necessitates rigorous validations through large multicenter prospective studies.

\section{Acknowledgments}

This work was supported by the Science and Technology Program of Guangzhou, China (grant number 201607010117). We thank Dr Flora Han-Zhang from Burning Rock Biotech for editing and valuable discussion.

\section{Disclosure}

The authors report no conflicts of interest in this work.

\section{References}

1. Goldstraw P, Crowley J, Chansky K, et al. The IASLC lung cancer staging project: proposals for the revision of the TNM stage groupings in the forthcoming (seventh) edition of the TNM classification of malignant tumours. $J$ Thorac Oncol. 2007;2(8):706-714.

2. Siegel RL, Miller KD, Jemal A. Cancer statistics, 2015. CA Cancer $J$ Clin. 2015;65(1):5-29.

3. National Lung Screening Trial Research Team; Aberle DR, Adams AM, et al. Reduced lung-cancer mortality with low-dose computed tomographic screening. N Engl J Med. 2011;365(5):395-409.

4. Henschke CI. International Early Lung Cancer Action Program Investigators. Survival of patients with clinical stage I lung cancer diagnosed by computed tomography screening for lung cancer. Clin Cancer Res. 2007;13(17):4949-4950.

5. Gohagan JK, Marcus PM, Fagerstrom RM, et al. Final results of the Lung Screening Study, a randomized feasibility study of spiral CT versus chest X-ray screening for lung cancer. Lung Cancer. 2005;47(1):9-15.

6. Bach PB, Mirkin JN, Oliver TK, et al. Benefits and harms of CT screening for lung cancer: a systematic review. JAMA. 2012;307(22): 2418-2429.

7. de Koning HJ, Meza R, Plevritis SK, et al. Benefits and harms of computed tomography lung cancer screening strategies: a comparative modeling study for the U.S. Preventive Services Task Force. Ann Intern Med. 2014;160(5):311-320.

8. Croswell JM, Baker SG, Marcus PM, Clapp JD, Kramer BS. Cumulative incidence of false-positive test results in lung cancer screening: a randomized trial. Ann Intern Med. 2010;152(8):505-512, W176-W580.

9. Crowley E, Di Nicolantonio F, Loupakis F, Bardelli A. Liquid biopsy: monitoring cancer-genetics in the blood. Nat Rev Clin Oncol. 2013;10(8): 472-484.

10. Wan JC, Massie C, Garcia-Corbacho J, et al. Liquid biopsies come of age: towards implementation of circulating tumour DNA. Nat Rev Cancer. 2017;17(4):223-238.

11. Alix-Panabieres C, Pantel K. Clinical applications of circulating tumor cells and circulating tumor DNA as liquid biopsy. Cancer Discov. 2016; 6(5):479-491.

12. Haber DA, Velculescu VE. Blood-based analyses of cancer: circulating tumor cells and circulating tumor DNA. Cancer Discov. 2014;4(6): 650-661.

13. Bettegowda C, Sausen M, Leary RJ, et al. Detection of circulating tumor DNA in early- and late-stage human malignancies. Sci Transl Med. 2014;6(224):224ra224.

14. Plaks V, Koopman CD, Werb Z. Cancer. Circulating tumor cells. Science. 2013;341(6151):1186-1188.

15. Chaffer CL, Weinberg RA. A perspective on cancer cell metastasis. Science. 2011;331(6024):1559-1564.

16. Alix-Panabieres C, Pantel K. Circulating tumor cells: liquid biopsy of cancer. Clin Chem. 2013;59(1):110-118.

17. Pantel K, Alix-Panabieres C. Circulating tumour cells in cancer patients: challenges and perspectives. Trends Mol Med. 2010;16(9):398-406.

18. Mego M, Mani SA, Cristofanilli M. Molecular mechanisms of metastasis in breast cancer clinical applications. Nat Rev Clin Oncol. 2010; 7(12):693-701.

19. Allard WJ, Matera J, Miller MC, et al. Tumor cells circulate in the peripheral blood of all major carcinomas but not in healthy subjects or patients with nonmalignant diseases. Clin Cancer Res. 2004;10(20): 6897-6904.

20. Cristofanilli M, Budd GT, Ellis MJ, et al. Circulating tumor cells, disease progression, and survival in metastatic breast cancer. $N$ Engl $J$ Med. 2004;351(8):781-791.

21. Coumans F, Terstappen L. Detection and characterization of circulating tumor cells by the cellSearch approach. Methods Mol Biol. 2015; 1347:263-278.

22. Hanssen A, Wagner J, Gorges TM, et al. Characterization of different CTC subpopulations in non-small cell lung cancer. Sci Rep. 2016;6: 28010. 
23. Bednarz-Knoll N, Alix-Panabieres C, Pantel K. Plasticity of disseminating cancer cells in patients with epithelial malignancies. Cancer Metastasis Rev. 2012;31(3-4):673-687.

24. Wong MP. Circulating tumor cells as lung cancer biomarkers. J Thorac Dis. 2012;4(6):631-634.

25. Isobe K, Hata Y, Kobayashi K, et al. Clinical significance of circulating tumor cells and free DNA in non-small cell lung cancer. Anticancer Res. 2012;32(8):3339-3344.

26. Hirose T, Murata Y, Oki Y, et al. Relationship of circulating tumor cells to the effectiveness of cytotoxic chemotherapy in patients with metastatic non-small-cell lung cancer. Oncol Res. 2012;20(2-3):131-137.

27. Wang J, Wang K, Xu J, Huang J, Zhang T. Prognostic significance of circulating tumor cells in non-small-cell lung cancer patients: a metaanalysis. PLoS One. 2013;8(11):e78070.

28. Hanssen A, Loges S, Pantel K, Wikman H. Detection of circulating tumor cells in non-small cell lung cancer. Front Oncol. 2015;5:207.

29. Krebs MG, Sloane R, Priest L, et al. Evaluation and prognostic significance of circulating tumor cells in patients with non-small-cell lung cancer. J Clin Oncol. 2011;29(12):1556-1563.

30. Hofman V, Bonnetaud C, Ilie MI, et al. Preoperative circulating tumor cell detection using the isolation by size of epithelial tumor cell method for patients with lung cancer is a new prognostic biomarker. Clin Cancer Res. 2011;17(4):827-835.

31. Maheswaran S, Sequist LV, Nagrath S, et al. Detection of mutations in EGFR in circulating lung-cancer cells. $N$ Engl J Med. 2008; 359(4):366-377.

32. Punnoose EA, Atwal S, Liu W, et al. Evaluation of circulating tumor cells and circulating tumor DNA in non-small cell lung cancer: association with clinical endpoints in a phase II clinical trial of pertuzumab and erlotinib. Clin Cancer Res. 2012;18(8):2391-2401.

33. Punnoose EA, Atwal SK, Spoerke JM, et al. Molecular biomarker analyses using circulating tumor cells. PLoS One. 2010;5(9):e12517.

34. Kummar S, Ji J, Morgan R, et al. A phase I study of veliparib in combination with metronomic cyclophosphamide in adults with refractory solid tumors and lymphomas. Clin Cancer Res. 2012;18(6):1726-1734.

35. Wang LH, Pfister TD, Parchment RE, et al. Monitoring drug-induced gammaH2 AX as a pharmacodynamic biomarker in individual circulating tumor cells. Clin Cancer Res. 2010;16(3):1073-1084.

36. Ni X, Zhuo M, Su Z, et al. Reproducible copy number variation patterns among single circulating tumor cells of lung cancer patients. Proc Natl Acad Sci U S A. 2013;110(52):21083-21088.

37. Jahr S, Hentze H, Englisch S, et al. DNA fragments in the blood plasma of cancer patients: quantitations and evidence for their origin from apoptotic and necrotic cells. Cancer Res. 2001;61(4):1659-1665.

38. Pathak AK, Bhutani M, Kumar S, Mohan A, Guleria R. Circulating cell-free DNA in plasma/serum of lung cancer patients as a potential screening and prognostic tool. Clin Chem. 2006;52(10):1833-1842.

39. Lui YY, Chik KW, Chiu RW, Ho CY, Lam CW, Lo YM. Predominant hematopoietic origin of cell-free DNA in plasma and serum after sexmismatched bone marrow transplantation. Clin Chem. 2002;48(3): 421-427.

40. El Messaoudi S, Rolet F, Mouliere F, Thierry AR. Circulating cell free DNA: preanalytical considerations. Clin Chim Acta. 2013;424: 222-230.

41. Warton K, Lin V, Navin T, et al. Methylation-capture and nextgeneration sequencing of free circulating DNA from human plasma. BMC Genomics. 2014;15:476.

42. Schwarzenbach H, Alix-Panabieres C, Muller I, et al. Cell-free tumor DNA in blood plasma as a marker for circulating tumor cells in prostate cancer. Clin Cancer Res. 2009;15(3):1032-1038.

43. Shaw JA, Brown J, Coombes RC, et al. Circulating tumor cells and plasma DNA analysis in patients with indeterminate early or metastatic breast cancer. Biomark Med. 2011;5(1):87-91.

44. Schwarzenbach H, Hoon DS, Pantel K. Cell-free nucleic acids as biomarkers in cancer patients. Nat Rev Cancer. 2011;11(6):426-437.

45. Thakur BK, Zhang H, Becker A, et al. Double-stranded DNA in exosomes: a novel biomarker in cancer detection. Cell Res. 2014;24(6): 766-769.
46. Ramirez JL, Rosell R, Taron M, et al. 14-3-3sigma methylation in pretreatment serum circulating DNA of cisplatin-plus-gemcitabinetreated advanced non-small-cell lung cancer patients predicts survival: The Spanish Lung Cancer Group. J Clin Oncol. 2005;23(36): 9105-9112.

47. Balgkouranidou I, Chimonidou M, Milaki G, et al. SOX17 promoter methylation in plasma circulating tumor DNA of patients with non-small cell lung cancer. Clin Chem Lab Med. 2016;54(8):1385-1393.

48. Konecny M, Markus J, Waczulikova I, et al. The value of SHOX2 methylation test in peripheral blood samples used for the differential diagnosis of lung cancer and other lung disorders. Neoplasma. 2016;63(2): 246-253.

49. Zhang Y, Wang R, Song H, et al. Methylation of multiple genes as a candidate biomarker in non-small cell lung cancer. Cancer Lett. 2011; 303(1):21-28.

50. Diehl F, Schmidt K, Choti MA, et al. Circulating mutant DNA to assess tumor dynamics. Nat Med. 2008;14(9):985-990.

51. Chan KC, Jiang P, Zheng YW, et al. Cancer genome scanning in plasma: detection of tumor-associated copy number aberrations, singlenucleotide variants, and tumoral heterogeneity by massively parallel sequencing. Clin Chem. 2013;59(1):211-224.

52. Cheng F, Su L, Qian C. Circulating tumor DNA: a promising biomarker in the liquid biopsy of cancer. Oncotarget. 2016;7(30):48832-48841.

53. Gao Y, Zhang K, Xi H, et al. Diagnostic and prognostic value of circulating tumor DNA in gastric cancer: a meta-analysis. Oncotarget. 2017;8(4):6330-6340.

54. Mao C, Yuan JQ, Yang ZY, Fu XH, Wu XY, Tang JL. Blood as a substitute for tumor tissue in detecting EGFR mutations for guiding EGFR TKIs treatment of nonsmall cell lung cancer: a systematic review and meta-analysis. Medicine (Baltimore). 2015;94(21):e775.

55. Fan G, Zhang K, Ding J, Li J. Prognostic value of EGFR and KRAS in circulating tumor DNA in patients with advanced non-small cell lung cancer: a systematic review and meta-analysis. Oncotarget. 2017; 8(20):33922-33932

56. Klco JM, Miller CA, Griffith M, et al. Association between mutation clearance after induction therapy and outcomes in acute myeloid leukemia. JAMA. 2015;314(8):811-822.

57. Pecuchet N, Zonta E, Didelot A, et al. Base-position error rate analysis of next-generation sequencing applied to circulating tumor DNA in non-small cell lung cancer: A Prospective Study. PLoS Med. 2016; 13(12):e1002199.

58. Fujiwara K, Fujimoto N, Tabata M, et al. Identification of epigenetic aberrant promoter methylation in serum DNA is useful for early detection of lung cancer. Clin Cancer Res. 2005;11(3):1219-1225.

59. Shames DS, Girard L, Gao B, et al. A genome-wide screen for promoter methylation in lung cancer identifies novel methylation markers for multiple malignancies. PLoS Med. 2006;3(12):e486.

60. Shivapurkar N, Gazdar AF. DNA methylation based biomarkers in non-invasive cancer screening. Curr Mol Med. 2010;10(2):123-132.

61. Kneip C, Schmidt B, Seegebarth A, et al. SHOX2 DNA methylation is a biomarker for the diagnosis of lung cancer in plasma. J Thorac Oncol. 2011;6(10):1632-1638.

62. Powrozek T, Krawczyk P, Nicos M, Kuznar-Kaminska B, BaturaGabryel H, Milanowski J. Methylation of the DCLK1 promoter region in circulating free DNA and its prognostic value in lung cancer patients. Clin Transl Oncol. 2016;18(4):398-404.

63. Powrozek T, Krawczyk P, Kucharczyk T, Milanowski J. Septin 9 promoter region methylation in free circulating DNA-potential role in noninvasive diagnosis of lung cancer: preliminary report. Med Oncol. 2014;31(4):917.

64. Ponomaryova AA, Rykova EY, Cherdyntseva NV, et al. Potentialities of aberrantly methylated circulating DNA for diagnostics and posttreatment follow-up of lung cancer patients. Lung Cancer. 2013;81(3): 397-403.

65. Balgkouranidou I, Chimonidou M, Milaki G, et al. Breast cancer metastasis suppressor-1 promoter methylation in cell-free DNA provides prognostic information in non-small cell lung cancer. Br J Cancer. 2014; 110(8):2054-2062. 
66. Wang H, Zhang B, Chen D, et al. Real-time monitoring efficiency and toxicity of chemotherapy in patients with advanced lung cancer. Clin Epigenetics. 2015;7:119.

67. Schmidt B, Beyer J, Dietrich D, Bork I, Liebenberg V, Fleischhacker M. Quantification of cell-free mSHOX2 Plasma DNA for therapy monitoring in advanced stage non-small cell (NSCLC) and small-cell lung cancer (SCLC) patients. PLoS One. 2015;10(2):e0118195.

68. Salazar F, Molina MA, Sanchez-Ronco M, et al. First-line therapy and methylation status of CHFR in serum influence outcome to chemotherapy versus EGFR tyrosine kinase inhibitors as second-line therapy in stage IV non-small cell lung cancer patients. Lung Cancer. 2011; 72(1):84-91.

69. Mitchell PS, Parkin RK, Kroh EM, et al. Circulating microRNAs as stable blood-based markers for cancer detection. Proc Natl Acad Sci US A. 2008;105(30):10513-10518.

70. Chen X, Ba Y, Ma L, et al. Characterization of microRNAs in serum: a novel class of biomarkers for diagnosis of cancer and other diseases. Cell Res. 2008;18(10):997-1006.

71. Fan L, Qi H, Teng J, et al. Identification of serum miRNAs by nanoquantum dots microarray as diagnostic biomarkers for early detection of non-small cell lung cancer. Tumour Biol. 2016;37(6):7777-7784.

72. Volinia S, Calin GA, Liu CG, et al. A microRNA expression signature of human solid tumors defines cancer gene targets. Proc Natl Acad Sci US A. 2006;103(7):2257-2261.

73. Boeri M, Verri C, Conte D, et al. MicroRNA signatures in tissues and plasma predict development and prognosis of computed tomography detected lung cancer. Proc Natl Acad Sci U S A. 2011;108(9): 3713-3718.

74. Shen J, Todd NW, Zhang H, et al. Plasma microRNAs as potential biomarkers for non-small-cell lung cancer. Lab Invest. 2011;91(4): 579-587.

75. Bianchi F, Nicassio F, Marzi M, et al. A serum circulating miRNA diagnostic test to identify asymptomatic high-risk individuals with early stage lung cancer. EMBO Mol Med. 2011;3(8):495-503.

76. Markou A, Sourvinou I, Vorkas PA, Yousef GM, Lianidou E. Clinical evaluation of microRNA expression profiling in non small cell lung cancer. Lung Cancer. 2013;81(3):388-396.

77. Ma J, Liu S, Wang Y. MicroRNA-21 and multiple myeloma: small molecule and big function. Med Oncol. 2014;31(8):94.

78. Yang M, Shen H, Qiu C, et al. High expression of miR-21 and miR-155 predicts recurrence and unfavourable survival in non-small cell lung cancer. Eur J Cancer. 2013;49(3):604-615.

79. Trang P, Wiggins JF, Daige CL, et al. Systemic delivery of tumor suppressor microRNA mimics using a neutral lipid emulsion inhibits lung tumors in mice. Mol Ther. 2011;19(6):1116-1122.

80. Thery C, Ostrowski M, Segura E. Membrane vesicles as conveyors of immune responses. Nat Rev Immunol. 2009;9(8):581-593.
81. Huotari J, Helenius A. Endosome maturation. EMBO J. 2011;30(17): 3481-3500.

82. van der Pol E, Boing AN, Harrison P, Sturk A, Nieuwland R. Classification, functions, and clinical relevance of extracellular vesicles. Pharmacol Rev. 2012;64(3):676-705.

83. Bobrie A, Colombo M, Raposo G, Thery C. Exosome secretion: molecular mechanisms and roles in immune responses. Traffic. 2011; 12(12):1659-1668

84. Reclusa P, Sirera R, Araujo A, et al. Exosomes genetic cargo in lung cancer: a truly Pandora's box. Transl Lung Cancer Res. 2016;5(5): 483-491.

85. Kahlert C, Melo SA, Protopopov A, et al. Identification of doublestranded genomic DNA spanning all chromosomes with mutated KRAS and p53 DNA in the serum exosomes of patients with pancreatic cancer. J Biol Chem. 2014;289(7):3869-3875.

86. Allenson K, Castillo J, San Lucas FA, et al. High prevalence of mutant KRAS in circulating exosome-derived DNA from early-stage pancreatic cancer patients. Ann Oncol. 2017;28(4):741-747.

87. Hao YX, Li YM, Ye M, et al. KRAS and BRAF mutations in serum exosomes from patients with colorectal cancer in a Chinese population. Oncol Lett. 2017;13(5):3608-3616.

88. Reclusa P, Giallombardo M, Castiglia M, et al. P2.06: Exosomal miRNA analysis in non-small cell lung cancer: new liquid biomarker?: track: biology and pathogenesis. J Thorac Oncol. 2016;11(10S):S219-S220.

89. Giallombardo M, Chacartegui Borras J, Castiglia M, et al. Exosomal miRNA analysis in non-small cell lung cancer (NSCLC) patients' plasma through qPCR: a feasible liquid biopsy tool. J Vis Exp. 2016;111.

90. Yu HA, Perez L, Chang Q, et al. A phase 1/2 trial of ruxolitinib and erlotinib in patients with EGFR-mutant lung adenocarcinomas with acquired resistance to erlotinib. J Thorac Oncol. 2017;12(1):102-109.

91. Jakobsen KR, Paulsen BS, Baek R, Varming K, Sorensen BS, Jorgensen MM. Exosomal proteins as potential diagnostic markers in advanced non-small cell lung carcinoma. J Extracell Vesicles. 2015;4: 26659.

92. Sandfeld-Paulsen B, Jakobsen KR, Baek R, et al. Exosomal proteins as diagnostic biomarkers in lung cancer. J Thorac Oncol. 2016;11(10): 1701-1710.

93. Molina R, Filella X, Auge JM, et al. Tumor markers (CEA, CA 125, CYFRA 21-1, SCC and NSE) in patients with non-small cell lung cancer as an aid in histological diagnosis and prognosis. Comparison with the main clinical and pathological prognostic factors. Tumour Biol. 2003;24(4):209-218.

94. Barlesi F, Gimenez C, Torre JP, et al. Prognostic value of combination of Cyfra 21-1, CEA and NSE in patients with advanced non-small cell lung cancer. Respir Med. 2004;98(4):357-362.
OncoTargets and Therapy

\section{Publish your work in this journal}

OncoTargets and Therapy is an international, peer-reviewed, open access journal focusing on the pathological basis of all cancers, potential targets for therapy and treatment protocols employed to improve the management of cancer patients. The journal also focuses on the impact of management programs and new therapeutic agents and protocols on
Dovepress

patient perspectives such as quality of life, adherence and satisfaction The manuscript management system is completely online and includes a very quick and fair peer-review system, which is all easy to use. Visit http://www.dovepress.com/testimonials.php to read real quotes from published authors. 